\title{
Color Duplex Ultrasound-Guided Foam Sclerotherapy: An Approach in the Management of Patients with Superficial Varicosities of Lower Extremity
} Gamal WM ${ }^{1 *}$ and Mohamed AS $^{2}$

${ }^{1}$ Department of Vascular Surgery, Qena Faculty of Medicine, South Valley University, Egypt ${ }^{2}$ Department of Vascular Surgery, Faculty of Medicine, Assiut University, Egypt

\begin{abstract}
Background: Duplex ultrasonography guided foam sclerotherapy is now considered a valuable option in varicose vein treatment; it is conducted as an outpatient procedure, does not require general anesthesia and compared with surgery results in an earlier return to normal activities. However, for foam treatment several sessions may be required.

Aim of work: The objective of this study is to describe the efficacy, results and safety of Ultrasound guided foam sclerotherapy (UGFS) for treating superficial venous disease of the lower limbs.

Patients and methods: 80 patients ( 28 males, 52 females) who were diagnosed to have clinical and radiological evidence of lower extremities venous diseases in the Department of Vascular Surgery at Qena and Assiut University Hospitals from November 2014 to November 2015 were included. Their ages ranged from 18 to 57 years. Local ethics committee approval and written informed consent were obtained.

As considered suitable for UGFS, the foam was prepared by Tessari's method. Any residual veins treated with another session.

Results: Eighty patients presenting with symptomatic varicose veins of superficial system. There were 52 females $(65 \%)$, and 28 males (35\%) with a mean age of $55.76 \pm 9.67$. CEAP grades of the patients were as follows; $(60.0 \%)$ in $\mathrm{C} 2,(10.0 \%)$ in C3, $(21.25 \%)$ in C4 $(2.5 \%)$ in C5 and $(6.25 \%)$ in C6. The affected segments of the superficial system which were treated were; $(70.0 \%)$ great saphenous, $(17.5 \%)$ small saphenous, $(6.25 \%)$ were great saphenous vein and varices and $(6.25 \%)$ were small saphenous vein and varices. The numbers of sessions needed to eradicate the affected segment were one session in $(70 \%)$, two sessions in $(18.75 \%)$ and three in $(11.25 \%)$. Minor complications encountered were skin discoloration in $30 \%$ of patients, superficial thrombophlebitis in $16 \%$ and an allergy to the foam sclerosant in $2.5 \%$. After one year follow up by colored duplex ultrasound (CDU) $(70 \%)$ had complete occlusion, $(15 \%)$ had partial occlusion and $(80 \%)$ of patients showed improvement of CEAP classification.

Conclusion: UGFS is a safe and effective treatment as an alternative to surgical treatment for superficial system varicosities. One and infrequently two to three treatment sessions, leads to complete eradication of superficial reflux in virtually $100 \%$ of cases. It is considered as an outpatient procedure. Complications are few, and appear mostly self-limiting.
\end{abstract}

Keywords: Varicose veins; Ultrasound guided foam sclerotherapy; Venous diseases

\section{Introduction}

Sclerotherapy is a medical procedure used to eliminate varicose veins and spider veins. Sclerotherapy involves an injection of a solution (generally a salt solution) directly into the vein. The solution irritates the lining of the blood vessel, causing it to swell and stick together, and the blood to clot.

A valuable treatment for primary varicose veins should be minimally invasive and capable of being used on primary and recurrent varicose veins so that it can be repeated as required. There should be few significant complications and the treatment should have good efficacy in abolishing venous reflux in saphenous trunks, perforating veins and varices [1]. The treatment should be accomplished at little cost and be capable of achieving both functional and cosmetic improvement with little time away from the patient's usual occupation [2]. Ultrasoundguided foam sclerotherapy has been considered particularly attractive because it avoids the need for general anesthesia, hospital admission and long recovery times [3]. The aim of this paper is to report the author's own series of patients treated by ultrasound guided foam sclerotherapy (UGFS) for the management of chronic venous disease.

\section{Methods}

\section{Patients}

Our group consisted of 80 patients ( 28 males, 52 females) who were diagnosed to have clinical and radiological evidence of lower extremities venous diseases in the Department of Vascular Surgery at Qena and Assiut University Hospitals from November 2014 to November 2015. Their ages ranged from 18 to 57 years. Local ethics committee approval and written informed consent were obtained. To

*Corresponding author: Gamal WM, Department of Vascular Surgery, Qena Faculty of Medicine, South Valley University, Qena, 83523, Egypt, Tel: 0020882146543; E-mail: walidgamal@yahoo.com

Received December 08, 2016; Accepted April 14, 2016; Published April 22, 2016

Citation: Gamal WM, Mohamed AS (2016) Color Duplex Ultrasound-Guided Foam Sclerotherapy: An Approach in the Management of Patients with Superficial Varicosities of Lower Extremity. J Vasc Med Surg 4: 263. doi:10.4172/23296925.1000263

Copyright: @ 2016 Gamal WM, et al. This is an open-access article distributed under the terms of the Creative Commons Attribution License, which permits unrestricted use, distribution, and reproduction in any medium, provided the original author and source are credited. 
Citation: Gamal WM, Mohamed AS (2016) Color Duplex Ultrasound-Guided Foam Sclerotherapy: An Approach in the Management of Patients with Superficial Varicosities of Lower Extremity. J Vasc Med Surg 4: 263. doi:10.4172/2329-6925.1000263

Page 2 of 5

be considered suitable for UGFS patients had to have symptomatic (CEAP C2-6) venous disease (i.e. treatment was not offered for cosmetic indications) and significant reflux ( $>0.5$ second) in a segment of superficial system as (Above knee Great saphenous vein (AK GSV), (below knee great saphenous vein (BK GSV), Short saphenous vein (SSV) and/or other superficial veins) on Duplex Ultrasound (DUS). Vein size was measured. Patients with absent pedal pulses or an ankle brachial pressure index $<0.9$ were excluded as were those with postthrombotic deep venous disease.

\section{Pre-treatment assessment}

History taking, clinical examination and DUS were done, at the initial clinic attendance in order to identify sites of superficial, deep and communicating venous reflux.

\section{UGFS treatment}

All treatments took less than $30 \mathrm{~min}$ and were performed as an office procedure in a duplex room. Sclerosant foam, prepared by Tessari's method using $1 \mathrm{~cm}^{3}$ sclerosing agent (aethoxysclerol 2\%) in one syringe, $3 \mathrm{~cm}^{3}$ of air in the other, connect to stopcock, apply 20 alternative movements from one syringe to the other through the stopcock and $4 \mathrm{~cm}^{3}$ of foam will soon be available giving $5 \mathrm{~cm}^{3}$ foam .

\section{Procedure}

- Mapping and drawing the venous network on skin choose the site(s) of injection; decide the section to be sclerosed.

- Preparing the skin.

- Placing a needle into vein under duplex guidance.

- Checking the blood reflux in hose, attaching needle to skin with adhesive tape.

- Preparing the foam.

- Positioning the probe over needle tip.

- Injecting the first bubbles.

- Verifying the bubbles inside the vein

- Injecting progressively the sclerosing foam, massage it with probe in the varicose network, check the foam fills all the desired veins.

- Checking the apparition of venous spasm.

- Removing needle, place a ball of cotton.

- Applying bandage and grade 2 medical stockings and keeping the stockings 24 hours, then all day long only.

- Follow up after 2 weeks either for (duplex evaluation or another injection). Figures 1-6 show our procedure.

Outcome measures and follow-up: The aim of treatment was to relieve the symptoms of venous hypertension, complete eradication of superficial venous reflux in the trunk and major tributaries of the superficial system.

All patients were seen at 1, 6 and 12 months after treatment in out patient's clinic. Repeated DUS was performed at each follow-up visit as the pre-treatment duplex. In addition, occlusion of the treated vein was assessed by a lack of compressibility and the absence of any flow. Complete occlusion was defined as occlusion over the entire length of the treated vein. Recanalization was defined as the presence of flow in either an ante grade or retrograde direction in a previously occluded vein. Where recanalization was found, the presence or absence of recurrent reflux was determined. Patients with residual reflux or recanalization at any follow-up appointment were offered further treatment by repeating foam sclerotherapy.

\section{Results}

80 patients with symptomatic varicose veins of superficial system were presented. There were 52 females (65\%), and 28 males (35\%) with

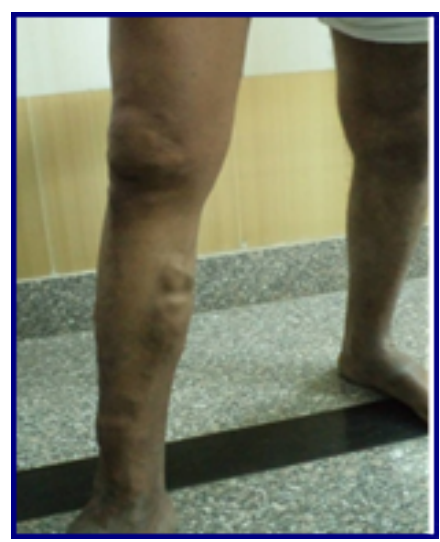

Figure 1: Rt 1ry vv of long saphenous v.

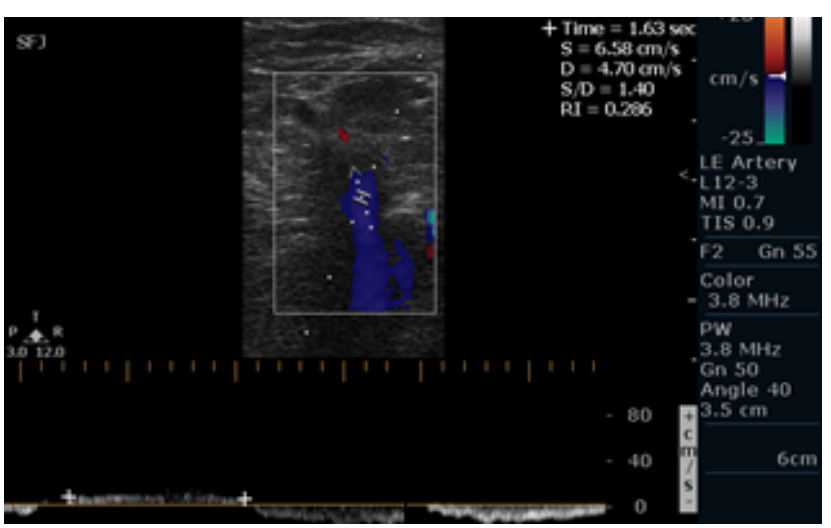

Figure 2: Reflux at SFJ by duplex.

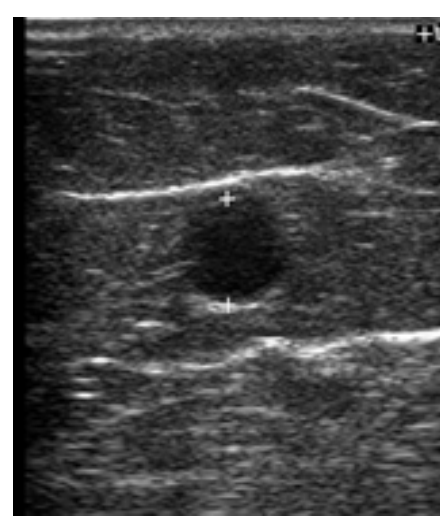

Figure 3: Diameter of right saphenous vein. 
Citation: Gamal WM, Mohamed AS (2016) Color Duplex Ultrasound-Guided Foam Sclerotherapy: An Approach in the Management of Patients with Superficial Varicosities of Lower Extremity. J Vasc Med Surg 4: 263. doi:10.4172/2329-6925.1000263

Page 3 of 5

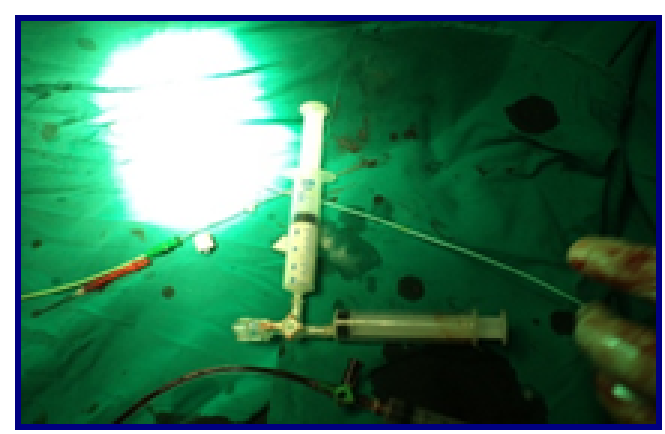

Figure 4: Foam formation.

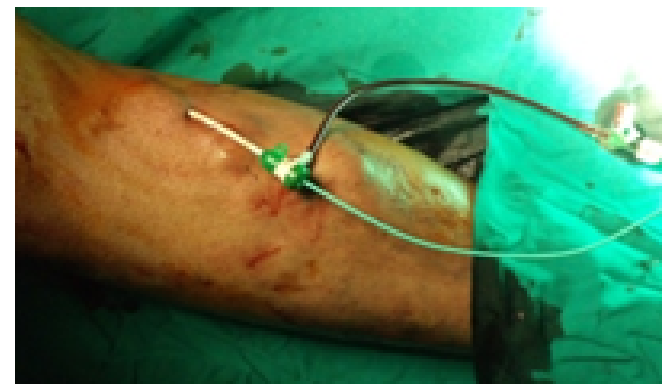

Figure 5: Sheath within the vein.

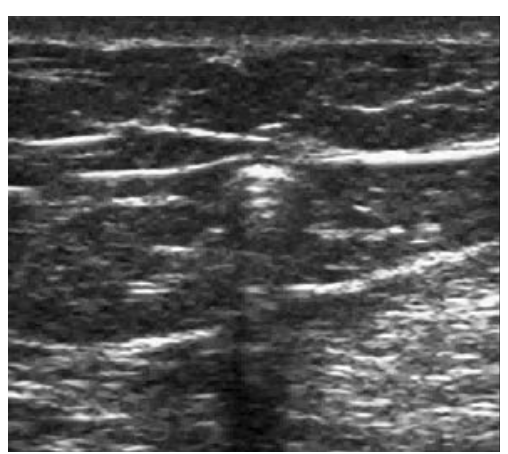

Figure 6: Diffusion of foam inside the vein.

a mean age of $55.76 \pm 9.67$. CEAP clinical grade shows $60 \%$ in C2, $10 \%$ in C3, 21.25\% in C4, 2.5\% in C5 and 6.25\% in C6 (Table 1). Etiology in the group was primary with $75 \%$ and secondary with $25 \%$. Anatomical patterns of venous reflux in cases were superficial and deep at $70 \%$ and superficial is only at $30 \%$. Pathophysiological classification in the group was $100 \%$ with reflux in all cases.

Different segments of the superficial system had been treated with duplex guided foam sclerotherapy: great saphenous with $70 \%$, small saphenous with $17.5 \%$, great saphenous vein and varcies with $6.25 \%$; small saphenous vein and varices with $6.25 \%$ (Table 2).

The number of sclerotherapy sessions. There were no visible VV in 56 legs (70\%) after one session and in 15 legs (18.75\%) after two treatment sessions resulting in both eradication of the reflux and disappearance of VV. Nine legs had residual VV after two sessions, but of them five were satisfied with the results and did not want further treatment. For the remaining four legs a further single session of foam injections directly into the visible varicosities successfully treated the residual VV (Table 3).

Reported complications with foam were; superficial thrombophlebitis in $16 \%$, pain in $15 \%$ and allergy in $2.5 \%$ of the patients (Table 4). Follow up with CDU: By 12 months, 56 patients (70\%) still had no visible VV or reflux after their primary course of treatment. Nine legs had recurrent VV in association with recanalization at 6 months, and another three had recurrent VV in association with recanalization at 12 months. Twelve patients were lost to follow-up (Table 5).

\section{Discussion}

Varicose veins represent a chronic, frequently relapsing, condition that develops secondary to valvular failure. It is, therefore, unrealistic to expect the complete and permanent eradication of superficial reflux in all patients following a single treatment whether that be surgical, UGFS, or another minimally invasive alternative [4].

Although still considered by many surgeons as the "gold standard" The effectiveness of GSV surgery is limited by the reluctance, based on fear of damaging the saphenous nerve, to strip the BK-GSV; a common cause of residual and recurrent disease. Furthermore, redo surgery for residual or recurrent reflux is usually difficult, often morbid and frequently associated with sub-optimal patient outcomes [5]. By contrast, as clearly demonstrated here, patients can be offered a primary course of UGFS treatments until all reflux has been eradicated. In most cases this requires only one treatment session using a modest volume of foam and is associated with a very low incidence of side

\begin{tabular}{|c|c|c|}
\hline S.No: & CEAP clinical grade & Descriptive \\
\hline 1 & C2 & $48(60.0 \%)$ \\
2 & C3 & $8(10.0 \%)$ \\
3 & C4 & $17(21.25 \%)$ \\
4 & C5 & $2(2.5 \%)$ \\
5 & C6 & $5(6.25 \%)$ \\
\hline
\end{tabular}

Table 1: Shows CEAP clinical grade in Foam Sclerotherapy group.

\begin{tabular}{|c|c|c|}
\hline S.No: & Item & Descriptive \\
\hline 1 & Great saphenous & $56(70.0 \%)$ \\
2 & Small saphenous & $14(17.5 \%)$ \\
3 & Great saphenous vein and varices & $5(6.25 \%)$ \\
4 & Small saphenous vein and varices & $5(6.25 \%)$ \\
\hline
\end{tabular}

Table 2: Shows veins treated in Foam sclerotherapy group.

\begin{tabular}{|c|c|}
\hline Number of sclerotherapy settings & Descriptive \\
\hline One & $56(70 \%)$ \\
Two & $15(18.75 \%)$ \\
More than two & $9(11.25 \%)$ \\
\hline
\end{tabular}

Table 3: Shows the number of Foam sclerotherapy sessions.

\begin{tabular}{|c|c|c|}
\hline S.No: & Item & Descriptive \\
\hline 1 & Superficial thrombophlebitis & $16(20 \%)$ \\
2 & Pain & $12(15.0 \%)$ \\
3 & Skin Staining & $24(30 \%)$ \\
4 & Deep vein thrombosis & 0.0 \\
5 & Alleregic reaction & $2(2.5 \%)$ \\
6 & Skin blistering & $4(5.0 \%)$ \\
7 & Visual disturbance & 0.0 \\
\hline
\end{tabular}

Table 4: Complications in foam sclerotherapy group.

\begin{tabular}{|c|c|c|}
\hline S.No: & Item & Descriptive \\
\hline 1 & Resolved complete occlusion & $56(70.0 \%)$ \\
2 & Resolved partial occlusion & $12(15.0 \%)$ \\
3 & CEAP declined & $64(80.0 \%)$ \\
\hline
\end{tabular}

Table 5: Follow up in Foam sclerotherapy group. 
effects and complications, rapid return to work and other activities. Furthermore, as also shown here, if recurrent reflux develops as result of recanalization that disease can be very simply and effectively treated, usually by a further single injection of foam. In our group CEAP clinical stages were 48 patients with $60 \%$ in C2, 8 with $10 \%$ in C3, 17 with $21.25 \%$ in C4, 2 with $2.5 \%$ in C5 and 5 with $6.25 \%$ in C6. The etiology in this group was $75 \%$ with primary (Ep) and $25 \%$ with secondary (Es). The authors Winterborn and colleagues [6] found that $100 \%$ with primary (Ep) and $0 \%$ with secondary (Es).

In present study anatomical pattern of venous reflux were superficial and deep was $70 \%$ and $30 \%$ with superficial cases only. Patho-physiological classification in the current group was $100 \%$ of cases reflux and $0 \%$ obstruction. In many literatures as with Darvall et al. study [7] of 91 legs, patients belong to C2 was $59 \%$, C3 was $4.5 \%, C 4$ was $23 \%$,C5 was $9 \%$ and C6 was $4.5 \%$ and $100 \%$ was primary in etiology and pathophysiology. Superficial and deep reflux accounts for $94.5 \%$ and superficial only at $5.5 \%$ treatment of GSV was done in $84.5 \%$ and of SSV in $15.5 \%$.

Rodrigo et al. [8] reported on 53 patients their classification was as follow $30.2 \%$ belong to $\mathrm{C} 2,30.2 \%$ to $\mathrm{C} 3,18.9 \%$ to $\mathrm{C} 4,11.3 \%$ to $\mathrm{C} 5$ and $9.4 \%$ to C6. According to anatomical classification $100 \%$ were superficial and also $100 \%$ were primary GSV treated in all patients.

In Wright et al. study [9] of 259 patients, $27 \%$ belong to C2, $46.33 \%$ to $\mathrm{C} 3,5.01 \%$ to $\mathrm{C} 4,8.88 \%$ to $\mathrm{C} 5$ and $12.74 \%$ to C6 and $100 \%$ with primary in etiology and pathophysiology. Superficial and deep reflux accounts for $92.66 \%$ and superficial was only $7.34 \%$. GSV intervention was done in $81.47 \%$ and SSV in $18.53 \%$.

The treated veins in the group were $70.0 \%$ with great saphenous, $17.5 \%$ with small saphenous, $6.25 \%$ with great saphenous vein and varices and $6.25 \%$ with small saphenous vein and varices. This agree with Thomasset et al. [10] who documented that $75.0 \%$ of treated veins was great saphenous, $13.0 \%$ of small saphenous, $8.0 \%$ with great saphenous vein and varices and $9.0 \%$ with small saphenous vein and varices.

Concerning efficacy, foam sclerotherapy appears to be efficacious treatment both for main trunk and minor vein disease. The results from our study revealed there were no visible VV in 56 legs (70\%) after one treatment session and in 15 legs (18.75\%) after two treatment sessions

Resulting in both eradication of the reflux and disappearance of their VV. Nine legs had residual VV after two sessions, but of them five were satisfied with the results and did not want further treatment. For the remaining four legs (5\%) a further single session of foam injections directly into the visible varicosities successfully treated the residual VV in the remaining four legs. These results were comparable with other studies as Darke and colleagues study [11] who treated 18 legs with UGFS; Ten legs (55.55\%) had complete occlusion after one treatment; a further five $(27.77 \%)$ had complete occlusion after two treatments. The three remaining legs had partial occlusion (either GSV still open but varicosities all closed or less than complete GSV occlusion but patient satisfied) after one, two or three treatments.

O'Hare and colleagues [12] study include 165 consecutive patients had foam sclerotherapy for truncal venous incompetence (91\%) of patients had a single treatment session, (9.09\%) required a second session and (1.21\%) patients needed 3 sessions to achieve target vein occlusion.

Of 27 patients underwent foam sclerotherapy in Figueiredo et al. study [13] three patients (11.11\%) underwent one session, 19 in $70.37 \%$ underwent two sessions and five patients (18.5\%) were treated during three sclerotherapy sessions. The average number of sessions per patient was 2.1.

In Darvall et al. study [7], Complete eradication of reflux in the entire (AK and BK) GSV was achieved in 84/91 (92\%) legs after one, and in a further 4/91 (4.5\%) legs after two treatment sessions (course of primary treatment). In three legs (3.5\%), complete eradication of GSV reflux was not achieved by one treatment session but these patients, despite residual GSV reflux, were content with the clinical result and declined further treatment sessions.

Concerning safety, serious adverse events including arterial events, pulmonary embolism, deep vein thrombosis, cutaneous necrosis and ulceration were statistically nil. The commonest adverse events associated with foam sclerotherapy in our study were skin discoloration in $(30 \%)$ of Patients, Superficial thrombophlebitis in (16\%) and an allergy to the foam sclerosant in $2.5 \%$ patients. Other series document various complications as Thomasset et al. study [10] who found that complications of UGFS were superficial thrombophlebitis $(18 \%$ of procedures), pain ( $14 \%$ of procedures), skin staining $(28 \%$ of procedures), deep vein thrombosis (DVT) ( $1 \%$ of procedures), allergic reaction ( $1 \%$ of procedures) and skin blistering ( $1 \%$ of procedures). A total of 48 patients experienced one, or more, of these complications. No patients experienced visual disturbance, a headache or other neurological symptoms.

In Myers et al. study [14] the only complication observed in this study was deep vein thrombosis which occurred in $3.2 \%$ of patients. This is somewhat higher than reported in other studies. In Coleridge study [15] the reported complications were as follow, thrombophlebitis occurred in a small number of patients (5\%) and was managed by analgesia, compression and aspiration of thrombus. Calf vein thrombosis was confined to isolated gastrocnemius veins or to part of the posterior tibial vein (1.23\%) All resolved with compression by stocking or bandage and exercise without use of anticoagulants. No major systemic complication such as anaphylaxis, stroke or transient ischemic attack occurred in this series. A number of patients (14,2\% of all patients treated) reported visual disturbance following treatment.

In present study the follow up with CDU was done at 6 months and 12 months. By 12 months, 56 (70\%) still had no visible VV or reflux after their primary course of treatment. Nine legs had recurrent $\mathrm{VV}$ in association with recanalization at 6 months, and another three had recurrent VV in association with recanalization at 12 months (12 were lost to follow-up). This agrees with Thomasset et al. study who found that the median timing of follow-up was 3 months (range 1.5-14 months) following treatment [10]. Duplex scans at follow-up revealed complete occlusion of the target vein following $79 \%$ of procedures $(n=100)$. Partial occlusion of the target vein was evident following $14 \%$ of procedures $(n=18)$ and a patent target vein was seen after $6 \%$ of procedures $(n=8)$. CEAP severity score declined in 123 patients following foam sclerotherapy and remained static in 3 patients.

In Darvall.et al. study [3] who found that by 12 months, 273/311 (87.8\%) still had no visible VV after their primary course of treatment ( 33 were lost to follow-up or had residual untreated VV). Six legs had recurrent $\mathrm{VV}$ in association with recanalisation at 6 months, and 19 had recurrent $\mathrm{VV}$ in association with recanalisation at 12 months. Fifteen of these 25 had further successful UGFS treatment resulting in both eradication of the reflux and disappearance of their recurrent VV. Ten legs had a few recurrent VV at 12 months but no recanalisation or reflux and only two of these needed further treatment; three had VV 
Citation: Gamal WM, Mohamed AS (2016) Color Duplex Ultrasound-Guided Foam Sclerotherapy: An Approach in the Management of Patients with Superficial Varicosities of Lower Extremity. J Vasc Med Surg 4: 263. doi:10.4172/2329-6925.1000263

Page 5 of 5

secondary to new reflux in the SSV. Also O'Hare et al. [12] found that; the treated vein was totally occluded in 68 legs $(74 \%)$, partially occluded in 9 with $10 \%$, and patent in 15 with $16 \%$. There was no significant difference in the occlusion rates in the different truncal veins.

In Coleridge study [15], 459 limbs have been reviewed at 6 months or more following treatment, average 11 months and range 6-46 months. This includes 363 of 886 GSVs and 141 of 263 SSVs. Duplex examinations of the GSVs showed occlusion had been obtained in 318 of 363 with $88 \%$. In the SSVs occlusion was present in 116 of 141 with $83 \%$. Darvall et al. study [7] found that of the 88 legs in which the primary course of UGFS achieved complete eradication of GSV reflux; recanalisation was observed in $1 / 79$ with $1.5 \%$ legs at 6 months and 9/77 with $12 \%$ legs at 12 months. Nine and 11 legs were not scanned at 6 and 12 months respectively.

\section{Conclusion}

Surgery does not provide a definitive treatment where UGFS is widely accepted as a treatment of primary venous incompetence (long and short saphenous), isolated incompetent saphenous tributaries, recurrent VV after surgery and patients with venous leg ulcers. UGFS has the advantages of being minimally invasive, can be repeated, patients return to work earlier and with few acceptable complications. One and infrequently two to three treatment sessions, leads to complete eradication of superficial reflux in virtually $100 \%$ of cases. It was considered as an outpatient procedure. Complications are few, and appear mostly self-limiting.

\section{References}

1. Guex JJ, Allaert FA, Gillet JL, Chleir F (2005) Immediate and midterm complications of sclerotherapy: report of a prospective multicenter registry of 1,173 sclerotherapy sessions. Dermatol Surg 31: 123-128.

2. Castro e Silva M, Cabral AL, Barros Jr N, Castro AA, ME Santos (2005) Diagnostic and treatment of venous disease Chronic: standards of guidance clinical the Brazilian Society of Angiology and Vascular Surgery (SBACV). J Vasc $\mathrm{Br}$ 4: S185-S194.

3. Darvall KA, Bate GR, Adam DJ, Bradbury AW (2009) Recovery, analgesia use, and return to normal activities after ultrasound-guided foam sclerotherapy compared with conventional surgery for varicose veins. Br J Surg 96: 12621267.

4. Kundu S, Lurie F, Millward SF, Padberg Jr F, Vedantham S, et al. (2007) Recommended reporting standards for endovenous ablation for the treatment of venous insufficiency: Joint statement of the American Venous Forum and the Society of Interventional Radiology. J Vasc Surg 46: 582-589.

5. Hayden A, Holdsworth J (2001) Complications following re-exploration of the groin for recurrent varicose veins. Ann R Coll Surg Engl 83: 272-273.

6. Winterborn RJ, Campbell WB, Heather BP, Earnshaw JJ (2004) The management of short saphenous varicose veins: a survey of the members of the vascular surgical society of Great Britain and Ireland. Eur J Vasc Endovasc Surg 28: $400-403$.

7. Darvall KA, Bate GR, Adam DJ, Silverman SH, Bradbury AW (2010) Duplex ultrasound outcomes following ultrasound-guided foam sclerotherapy of symptomatic primary great saphenous varicose veins. Eur J Vasc Endovasc Surg 40: 534-539.

8. Gonzalez-Zeh R, Armisen R, Barahona S (2008) Endovenous laser and echoguided foam ablation in great saphenous vein reflux: one-year follow-up results. Journal of Vascular Surgery 48: 940-946.

9. Wright $D$, Gobin JP, Bradbury AW, Coleridge-Smith $P$, Spoelstra H, et al. (2006) Varisolve polidocanol microfoam compared with surgery or sclerotherapy in the management of varicose veins in the presence of trunk vein incompetence. European randomized controlled trial Phlebology 21:180-190.

10. Thomasset SC, Butt Z, Liptrot S, Fairbrother BJ, Makhdoomi KR (2010) Ultrasound guided foam sclerotherapy: factors associated with outcomes and complications. Eur J Vasc Endovasc Surg 40: 389-392.

11. Darke SG, Baker SJ (2006) Ultrasound-guided foam sclerotherapy for the treatment of varicose veins. Br J Surg 93: 969-974.

12. O'Hare JL, Parkin D, Vandenbroeck CP, Earnshaw JJ (2008) Mid term results of ultrasound guided foam sclerotherapy for complicated and uncomplicated varicose veins. Eur J Vasc Endovasc Surg 36: 109-113.

13. Figueiredo M, Araújo S, Barros N Jr, Miranda F Jr (2009) Results of surgica treatment compared with ultrasound-guided foam sclerotherapy in patients with varicose veins: a prospective randomised study. Eur J Vasc Endovasc Surg 38: 758-763.

14. Myers KA, Jolley D, Clough A, Kirwan J (2007) Outcome of ultrasound-guided sclerotherapy for varicose veins: medium-term results assessed by ultrasound surveillance. Eur J Vasc Endovasc Surg 33: 116-121.

15. Smith PC (2006) Chronic venous disease treated by ultrasound guided foam sclerotherapy. Eur J Vasc Endovasc Surg 32: 577-583. 